\title{
Comportamento geodinamico
}

\author{
della Diga di Vodo di Cadore \\ (The geodinamical behaviour of the Dam of Vado di Catore)
}

P. F. BIAGI

Ricevuto il 20 settembre $1 ! 30$

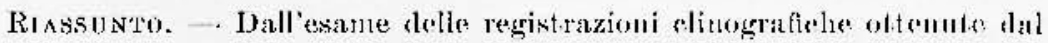

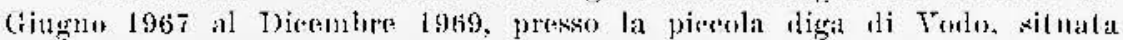
sul torrente Boite, affluente di destra del Piave, is emerso un importante fatto novo nell aubito tella microlinanica delle dirhe e cine che por lin. sorgere dellemta diurna in modo semsibile non o sempre necessario che mut

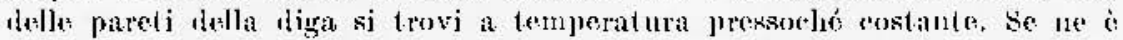
precisatu qui, su hase sperimentale, il camper di valinita.

Si e poi studiala la forma donda, in aleuni mesi, apporentemente nuovi tella componente sinistra destra e la si è collegala alle zone della diga illuminate dal solf: nelle varie ore tlel giorne.

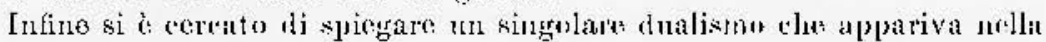
componente sinistra destra per quanto eneerne ogli spostamenti lunghi, derivanti dalle variazioni di temperatura metia.
\end{abstract}

Sumary. - The analysis of the elinegraplice resordings olut ained from June 1967 to December 1969, in the litile daun of Fonlo, siluated on the Boite river, riglit tribulary of the Piave, rovealed an important now fart about the dyuanies of the dams: it is net neessary that ont of the sines of the dam have a costant temperature to cause the arizing of the day-waves. Wo pronverl here on experimental hasis, when it is valit.

Then, we stulied, during sone motthis, tho wave - apparcnlly new of the left-right component: its shape is due to the sitles of the dam lighted with the sun in the different hours of the day.

Lastly, we tried fo explain the unuxual dualism apperaring in the left-right component, as regards the long displarements depending on the variations of the average temperature. 
lab digab di Voulo ì una diga di picoole dimensioni (altezan max $12 \mathrm{~m}$ ) siluatab sul torrente Roite, aflluente di destrab del Piave, nei pressi di Vodo di Carkore (Fig. 1).

In questa dira è in funzione una copplia di fotodinograli, uno per la registrazione di mieromovimenti in direzione monte valle as laltro fert la registrazione a $90^{\circ}$, tangenziabmente all'asse dolla diga, situati alla (quota foronamento, in eorrispondenza detla luce centitable del ponte.

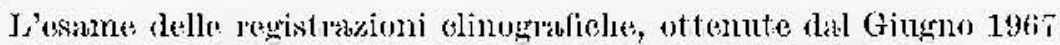
al Dicembre 1969, lan permesso di definire in modo esauriente l'origrine dei mieromovimenti che interessano la digas.

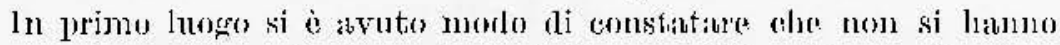
miromovimenti dovuti alle variazioni d'inviso. Di solito, in direzione monte valle, gli sbarmmenti risentono della spinta di pressione dovats alle variazioni del livello idrico. Si i però chiarito, in wu precedente

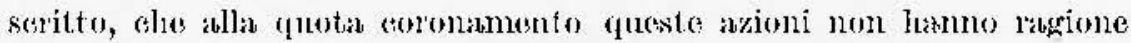
d'essere; i risultati ottenuti presso lab digat di Vordo, confermano quindi, vieppiù quanto è stato grià rilevato.

L'unica azione dovata all'invaso prod essere riscontrata nel fatio ahe, a bacino vouto, la diga $a$ piu sensibile (in modo apprasiscente in tirezione monte-valle) all azione dell'insolazione (vedere punto scomente) di quanto non lo sia a hacino pieno. Jat Figr. 2 serva da empio. Questo è in distocordo col fatto, rilevato altrove, che per l'insorgere dell'onda diurna in modo sensibile is nesessario ohe mob delle proteti della diga si

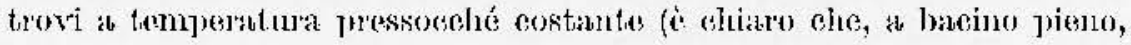
lab parete to monte dellab diga lab temperatima poeo variabiles).

Senza nulla togliore alla roridieitio inconfutabile di questio prineipio, lo studio attuale ha permesso di definime un limite: esso vale eick solo per dighe di grandi dimensioni. Per una diga some quellat di Vordo, piceolab e non struttumata a conei, l'insorgere dell'ondia diumba

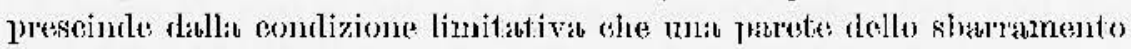
si trovi a temperatum pressocehe costatute eosi che, ab basino vuoto, mancando la spinta di pressione dolla massa d'andua che ne limita l'elasticitì, la diga è più sensibile alle azioni temiche, ameno in direziones monte-vialle.

P'assiamo ad un altion punto.

i) noto dagli studi esegutiti presso altre dighe ahe l"iusclabione is masab di micromovimenti. I'uscillazione diuma, notas sotto il nome di onda dinuru, e pabticolamente appariscente nolla diga di Volu. Se si considerano le Finnre da 3 as 1 t, ohe riproducono i obinogrammi ottenuti 


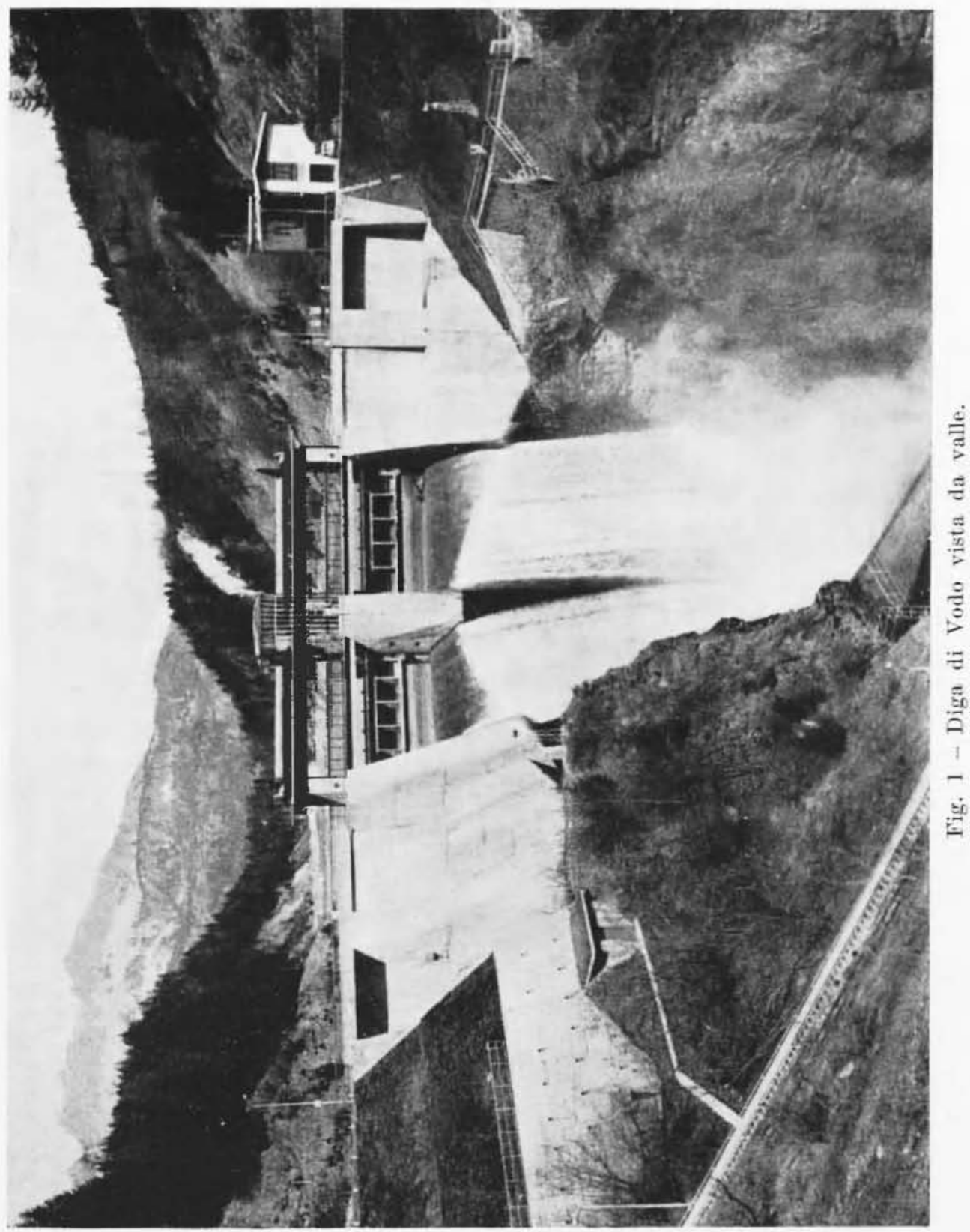




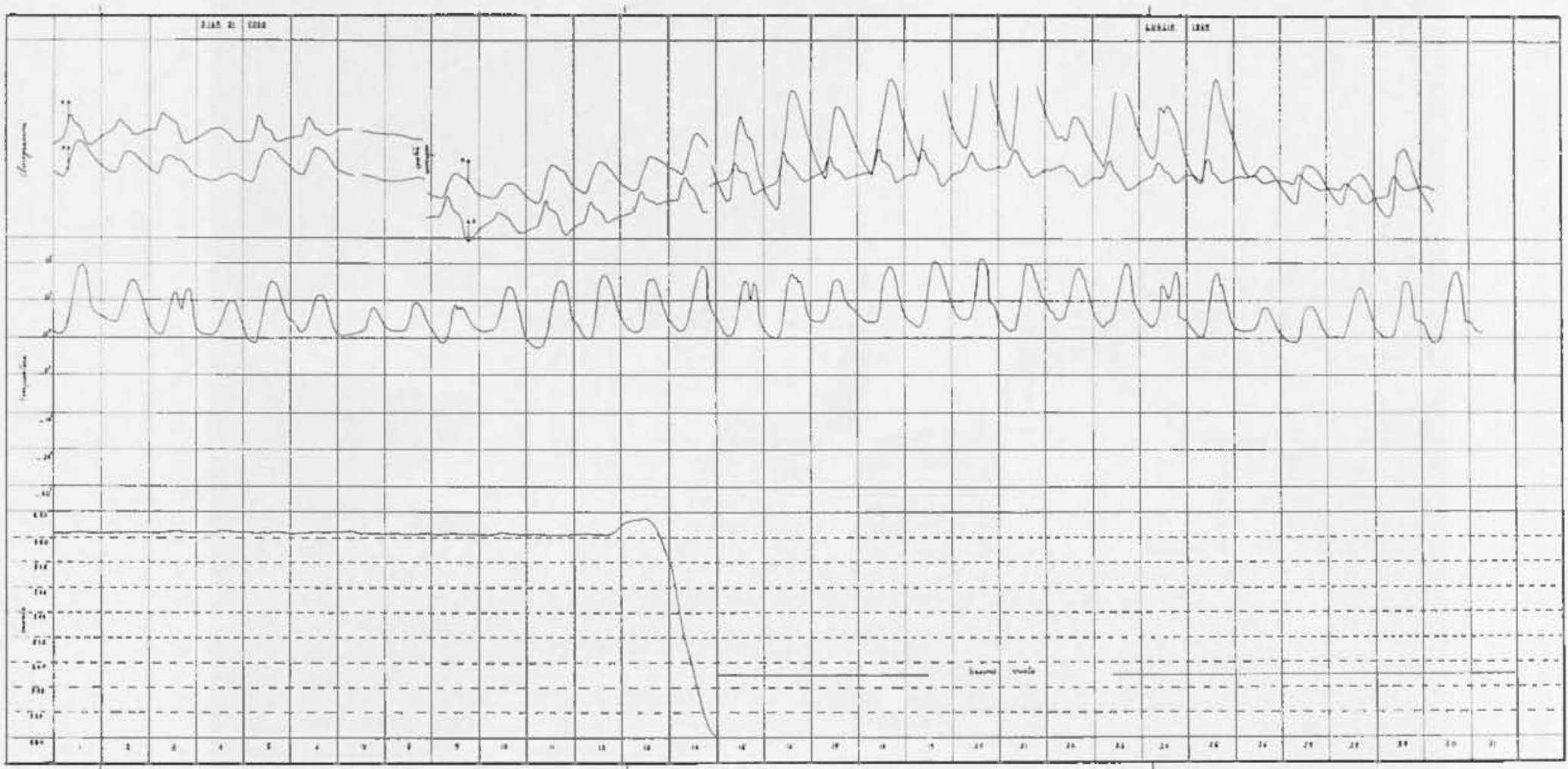

Fig. 2 - Diga di Vodo - Luglio 1967 - Si hamo esempi evidenti li onde diurne dopo lo svuotamento sel bacino. L'oseillazione diurna in direzione sinistra-lestra non mostra variazioni appariseenti. L'unda diurna della eomponente monte-valle risulta più ampia a bacino vuoto, pur exsendo le escursioni termiche slello stesso oriline di quelle a bacino pieno. 


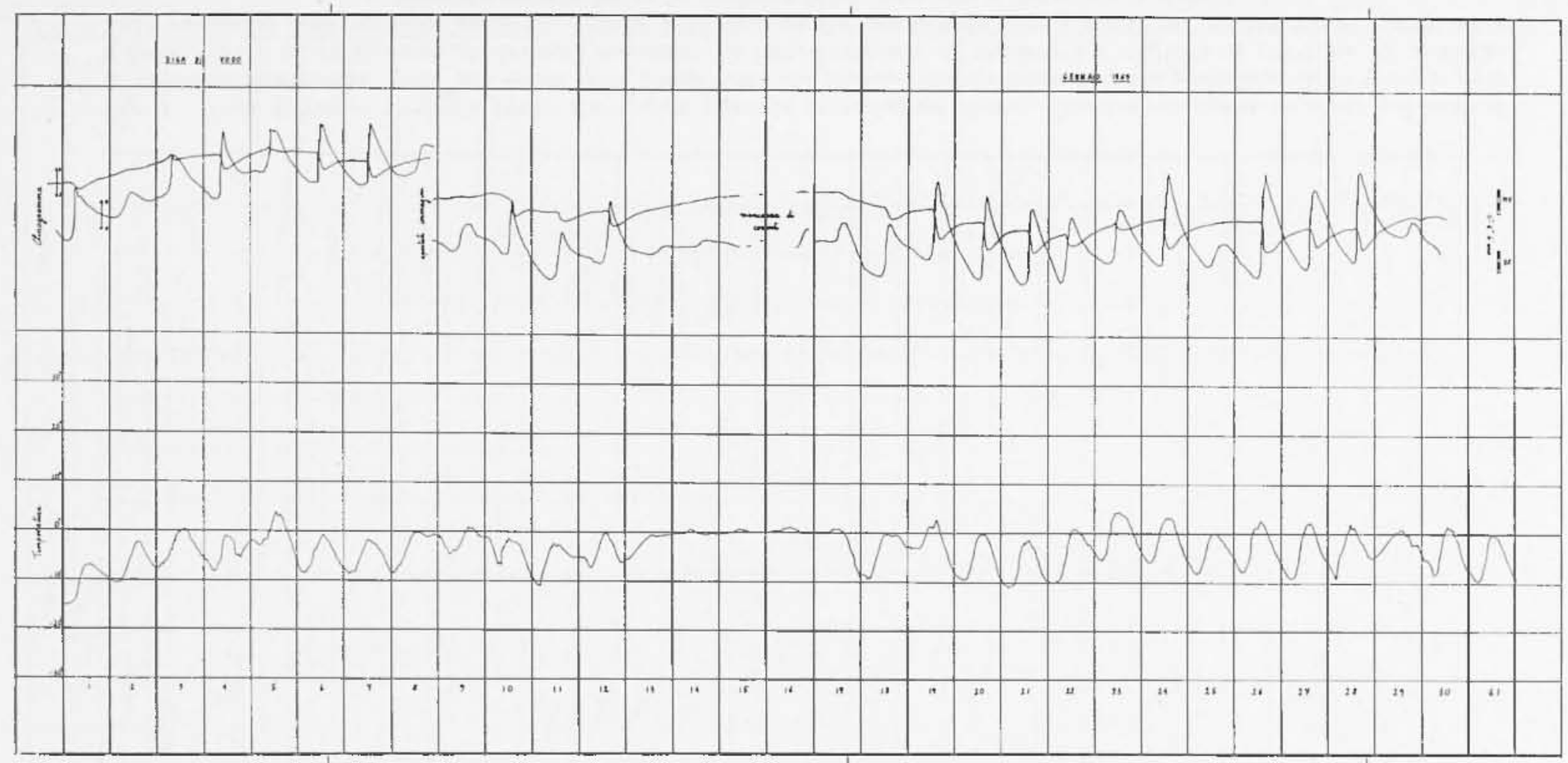

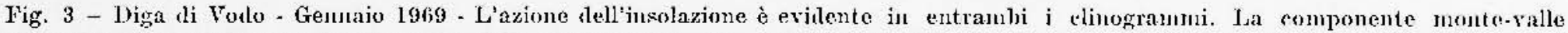
rispeechia sostanzialuente lanklumento della temperatura. Ja componente sinjstra-destra prosenta onde diurne a sinistrit, mentre le variazioni della temperatura metla dell'aria portano la diga a destra per risealdancuto, si veda ad esempio la risposta all'anmento terunico slall 1 al $\bar{j}$. 


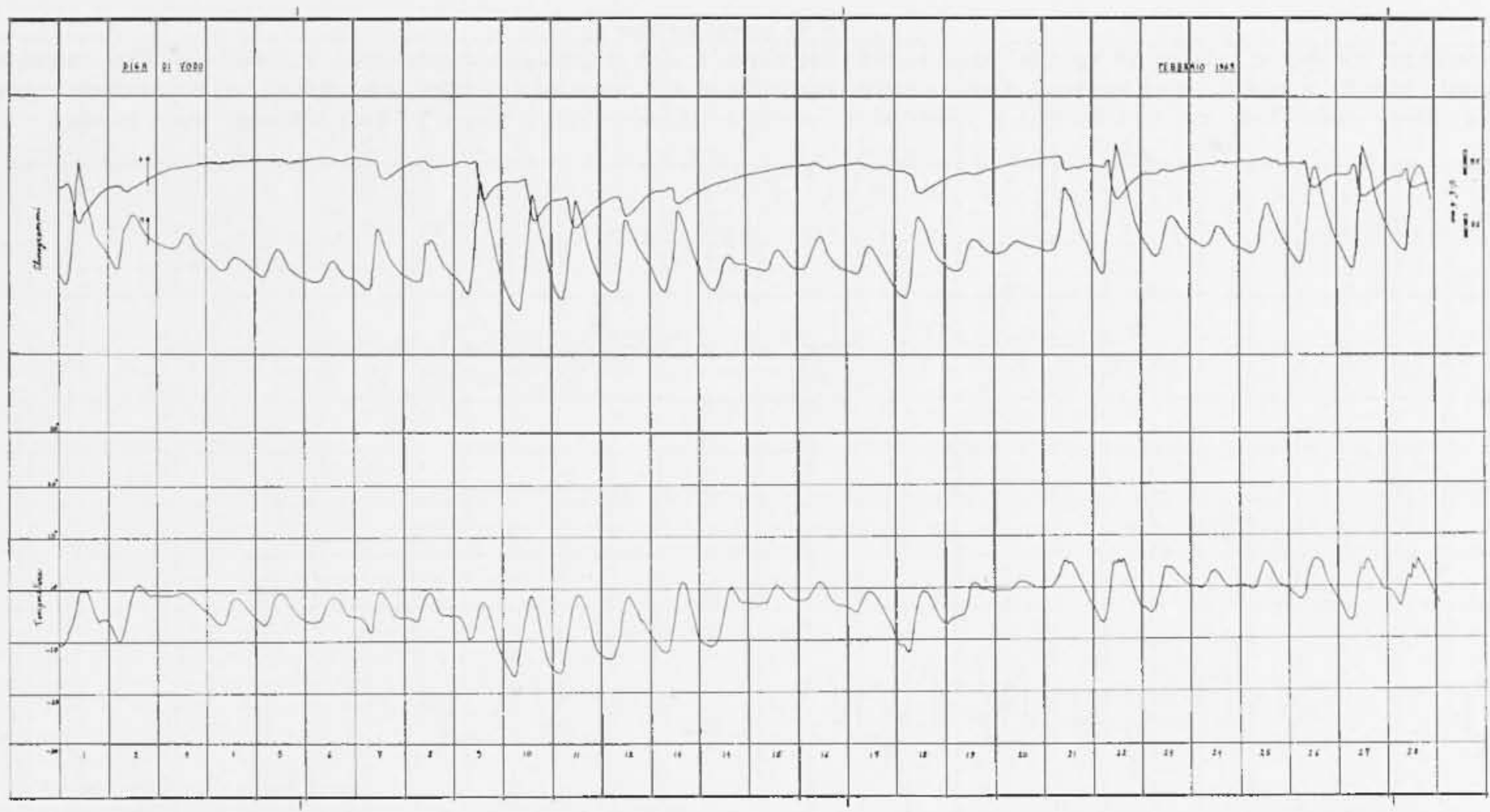

Fig. 4 - Diga di Vodo - Febbraio 1969 - Per quanto riguarda le variazioni diume, l'insolazione agisce in modo più vistoso in direzione monte-falle. Nello pur searse onde diurne della componente sinistra-destra fa gia la sua comparsa un picco iniziale a destra, che si andrà accentuando nei mesi successivi. J.e lente variazioni di temperatura dell'aria si riflettono in analoghe lente variazioni della verticale apparente; quelle della componente monte-valle sono a monte per riscaldamento, quelle della componente sinistra-destra in corrispondenza, sono ora a destra ora a sinistra. 


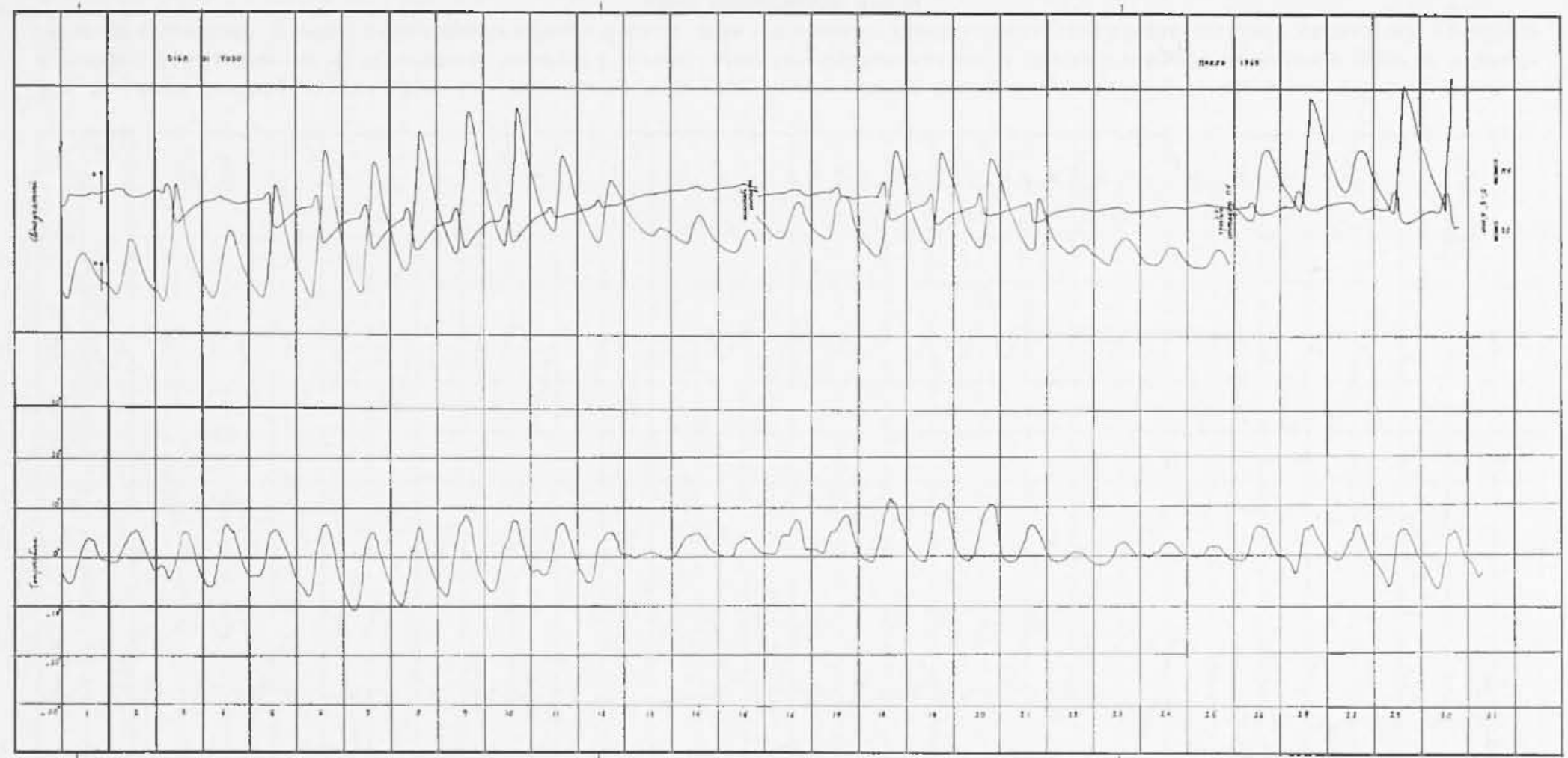

Fig. 5 - Diga di Vodo - Marzo 1969 - L'inizialo rotazione a destra nollo onde diurno della componente sinistra-destra è, in questo mese, ben netta. Notevole il parallolismo fra l'andamento tormico o lo variazioni della verticalo apparente della eomponente monte-valle. Le variazioni a lungo perioilo della componente sinistra-ilestra sono incerte per quanto concerne il vorso. 


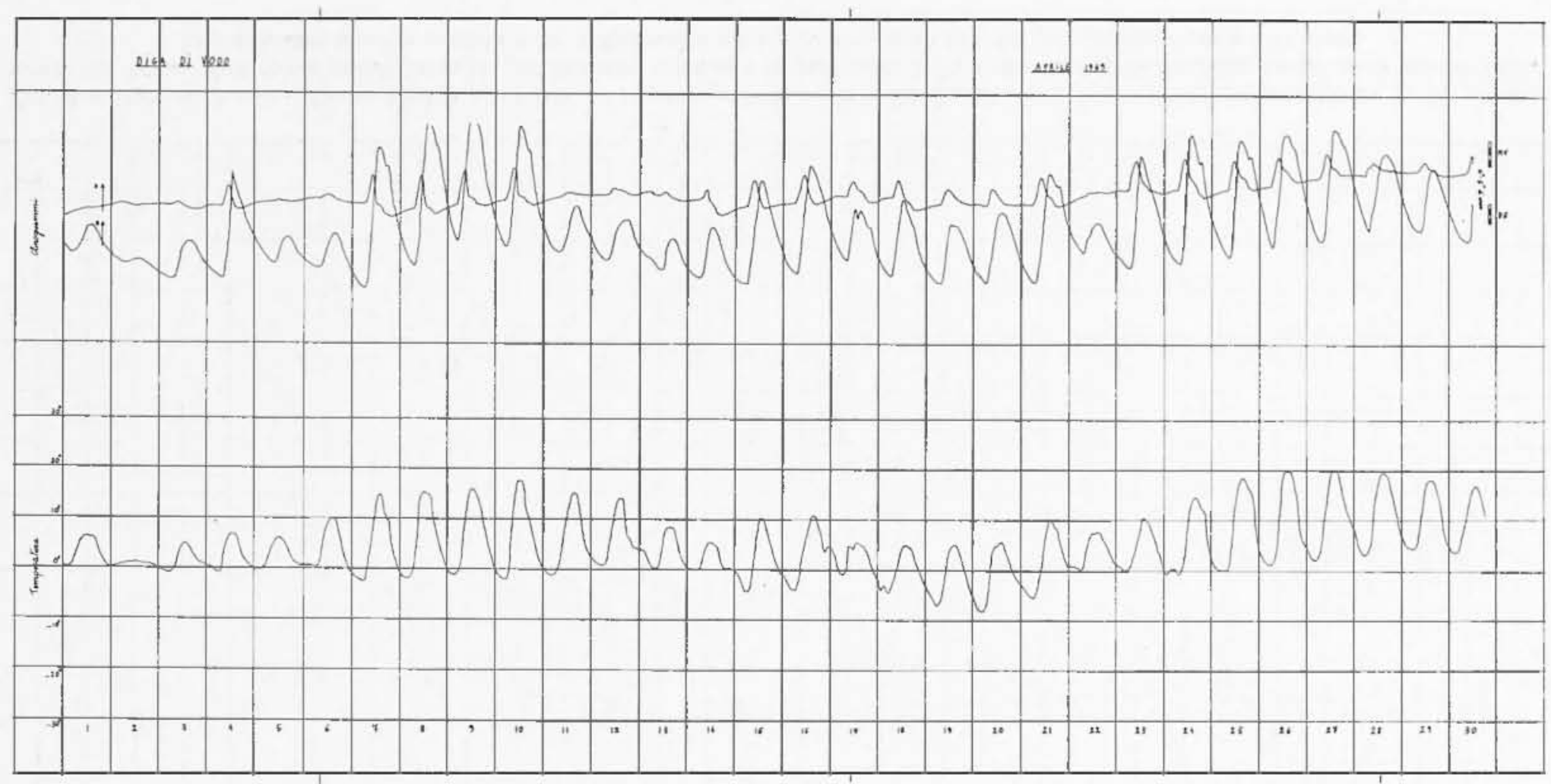

Fig. 6 - Diga di Vodo - Aprile 1969 . Te onde dime della componente sinistra-destra cominciano a presentate una ulteriore rotazione

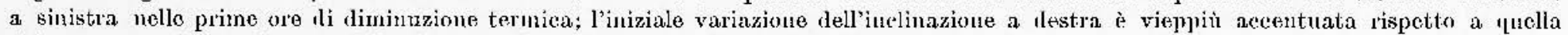
dol meso precenlente. Il verko della risposta a lumga durata, nella conponente sinistra-ilestra, non è legato in modo univoco alle variazioni ilella temperatura metlia. 


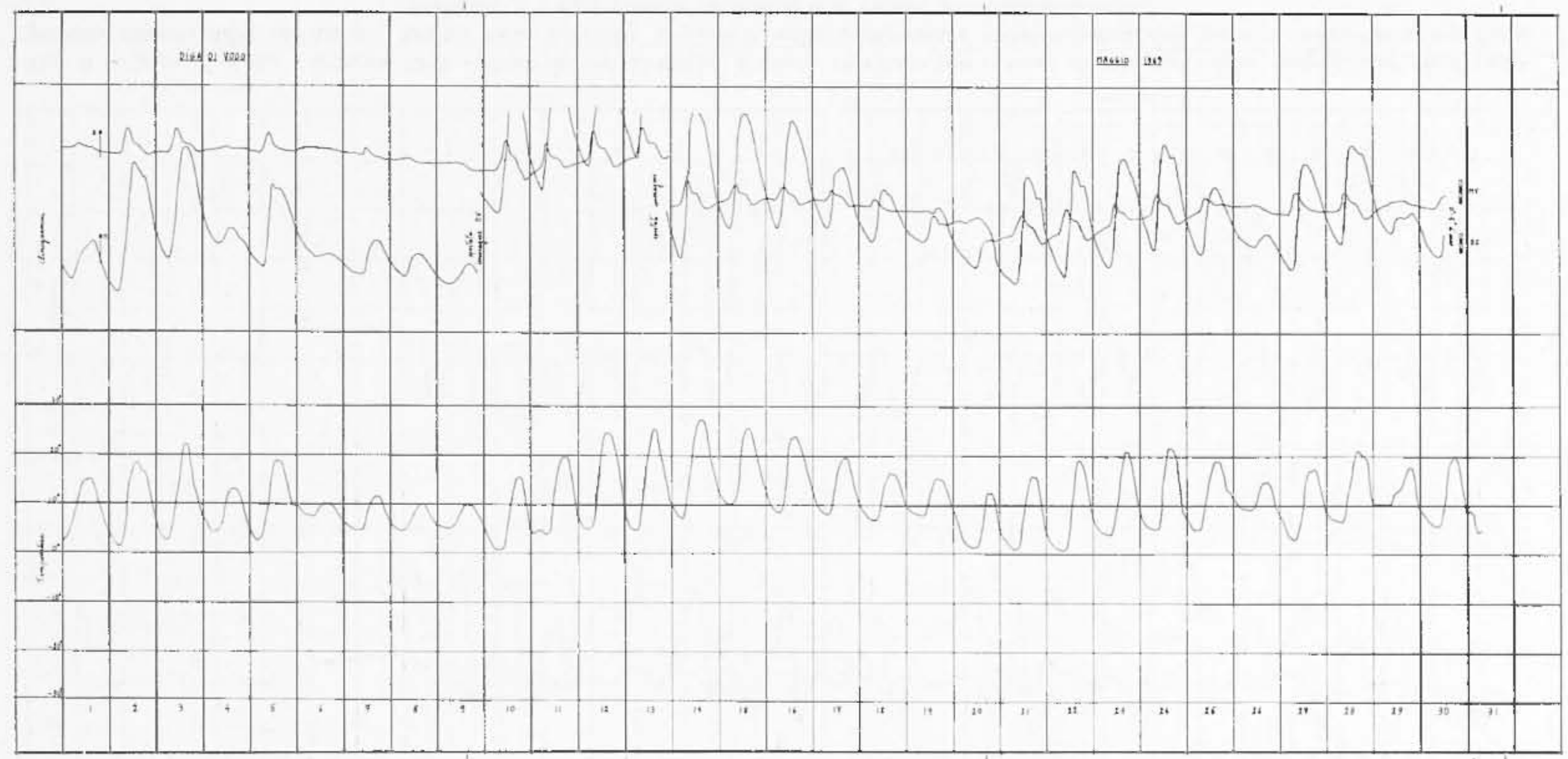

Fig. 7 - Diga di Vodo - Maggio 1969 - Presenza. nellonda diurna della componente sinistra-destra, di un piseo iniziale di rotazione a destra e di una coda a sinistra all'inizio della fase discondente della tomperatura. Notte onde diume nel elinogramma monte-valle. Contrasti accentuati in ambo le componenti dovnti alle variazioni della temperatura dollaria, che, in questo mese, provocano variazioni t'inclinazione a thestra e a monte, per riscaldamento. 


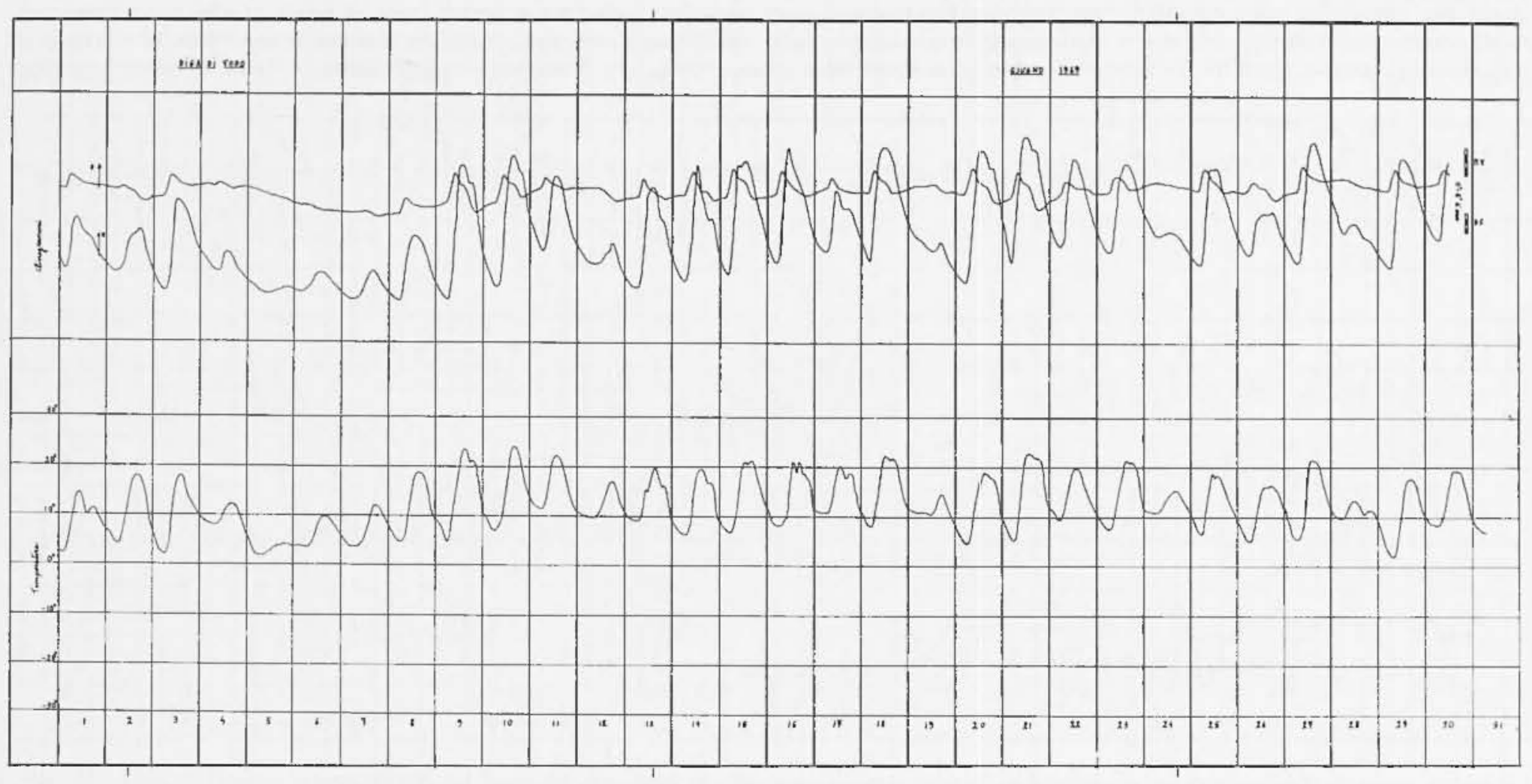

Fig. 8 - Diga di Vodo - Giugno 1969 - Osservazioni antaloghe a quelle riportate per il mese di Maggio: ampie onde diurne nella com. ponente monte-valle; oscillazioni diume della verticale apparente nella componente sinistra-destra con picco e coda; variazioni della verticale a lungo periodo a destra e a noonte per riscaldamento. 


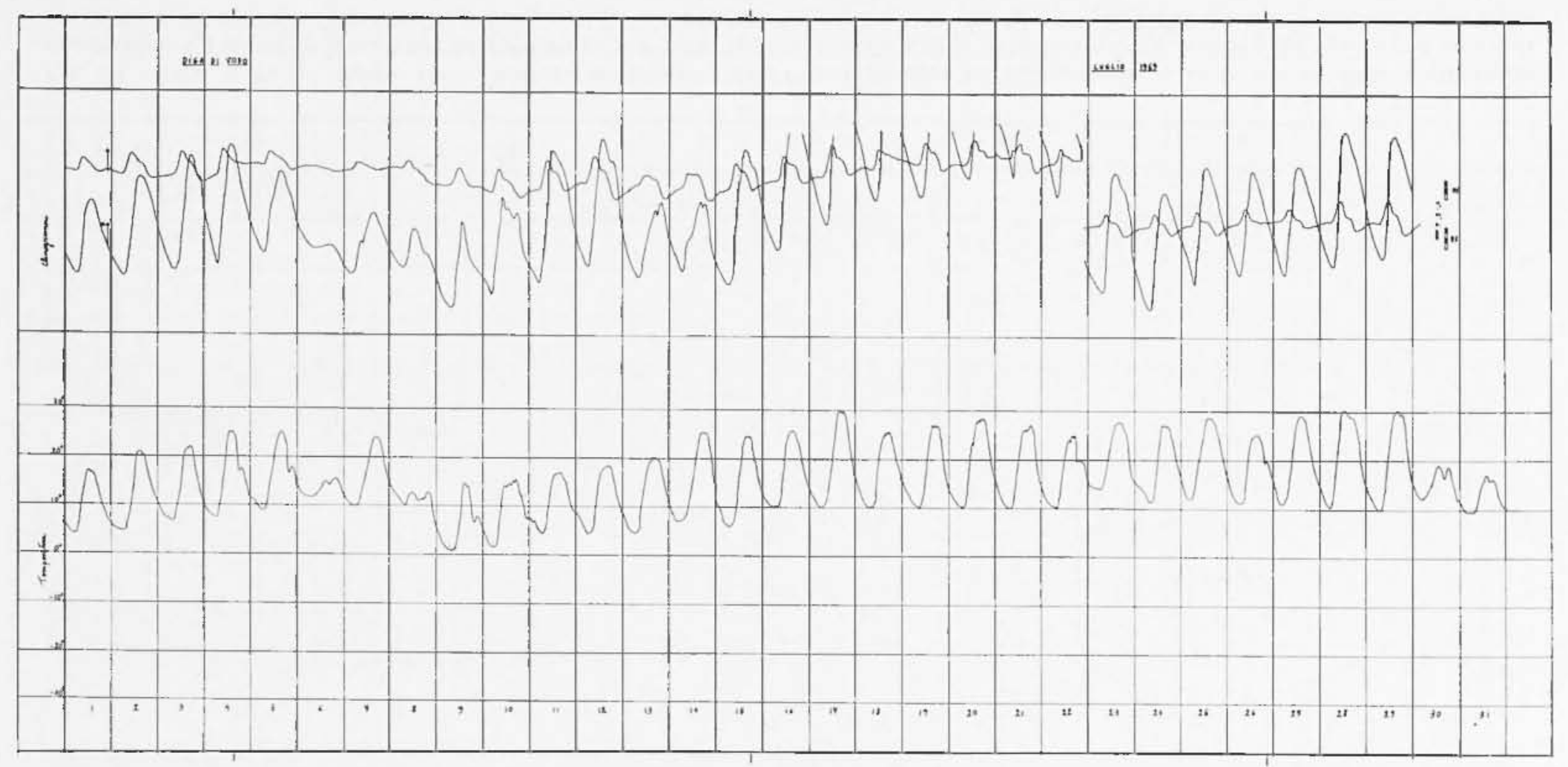

Fig. 9 - Diga di Vodo - Luglio 1969 - Le variazioni d'inclinazione a lungo periodo nella componente sinistra-destra sono generalmente

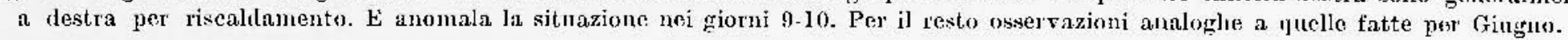




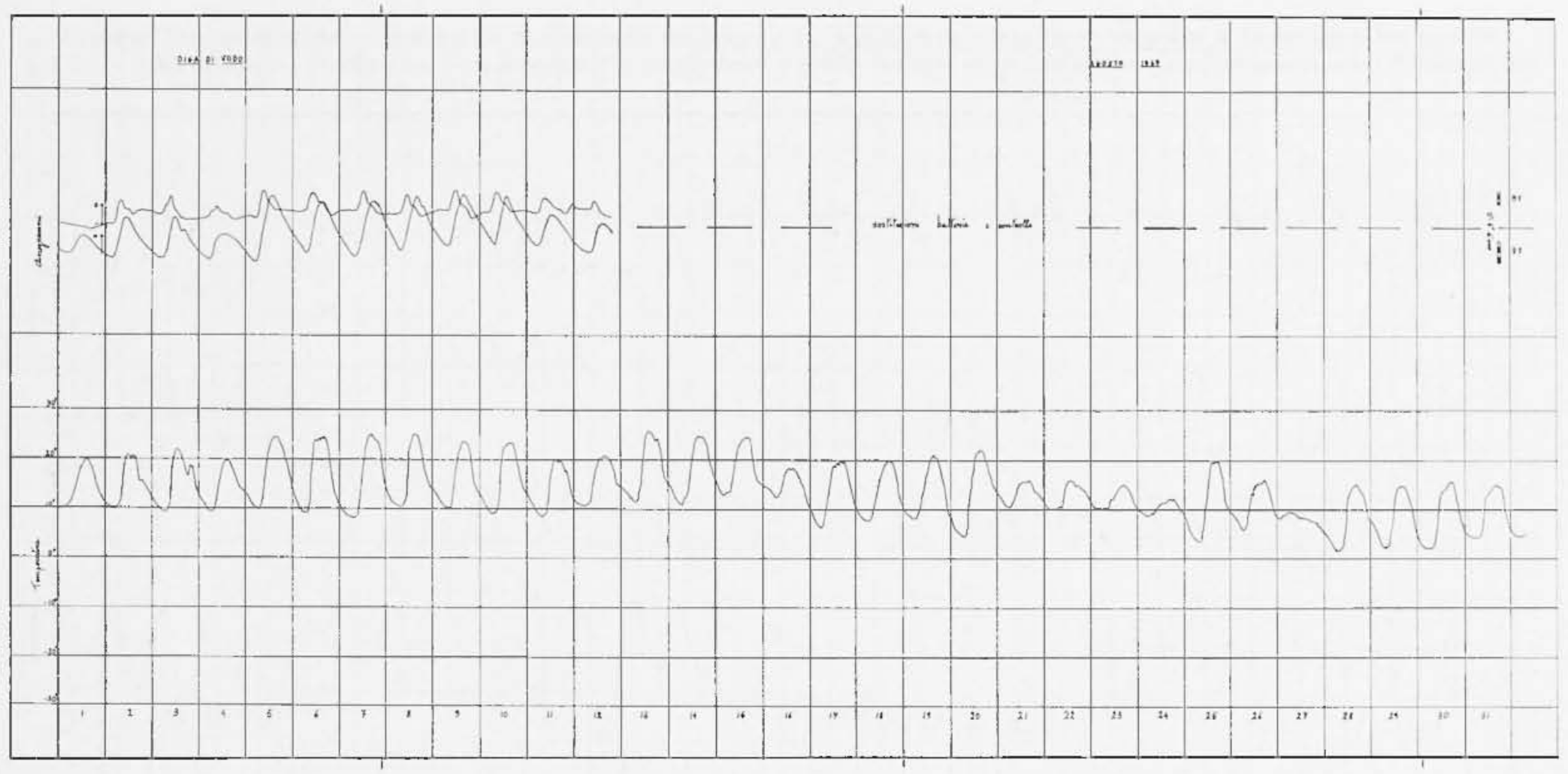

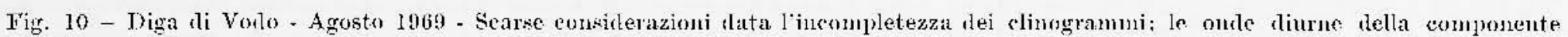
sinistra-destra presentano i earatleri in precedenza più volte rilevati; le onde diurne della componente monte-valle sono meno ampifs di melle del mese di Luglio, pur con escursioni temiche conparabili. Va perio notato che la corrispondenza tra mu e secondi d'areo varia da mese a mese. 


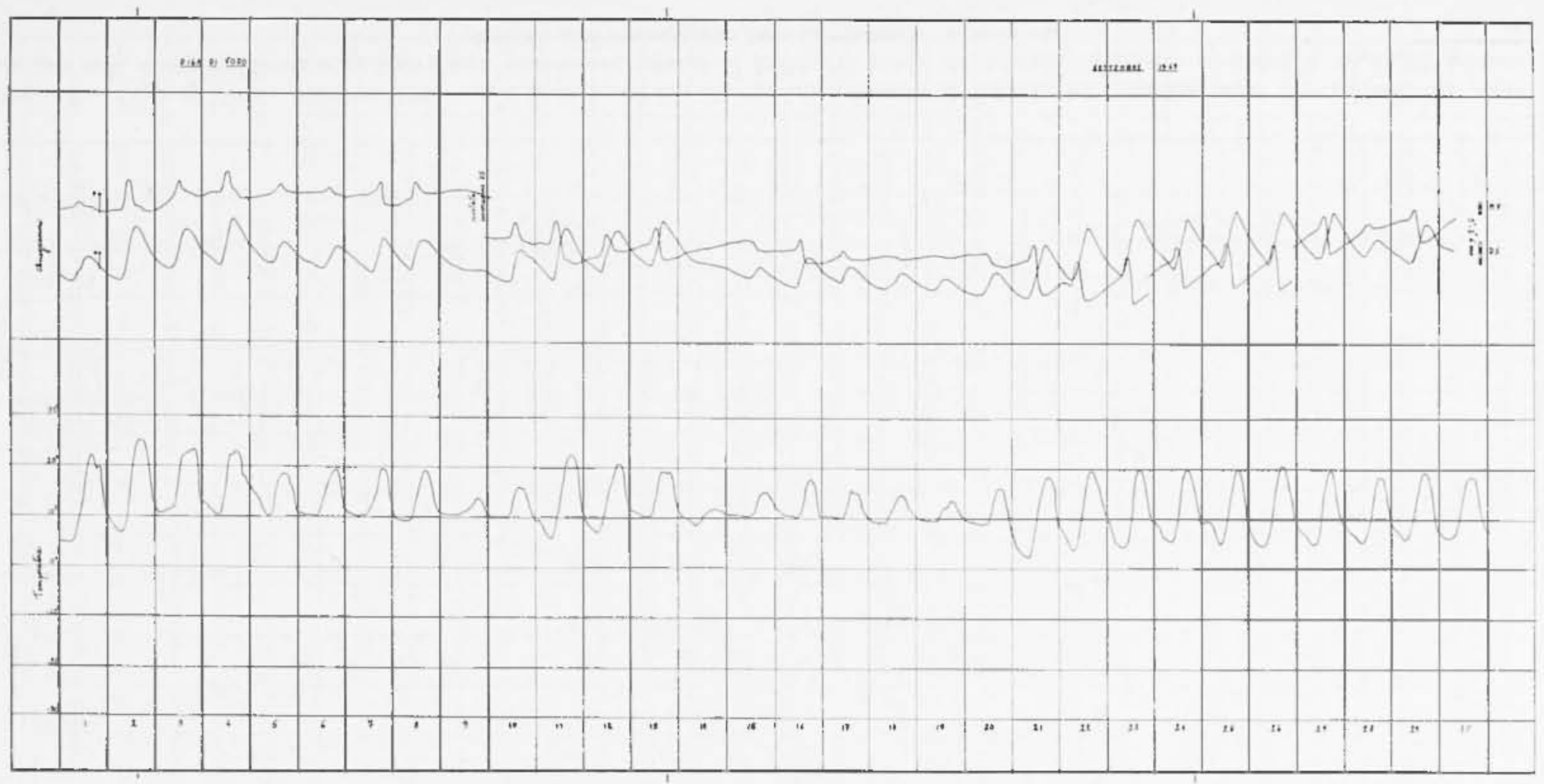

Fig. 11 - Diga di Vodo. Setlembre 1960 - Je onde diurne della componente sinistra-slestra. nellia seconda meti del mese, nou presen-

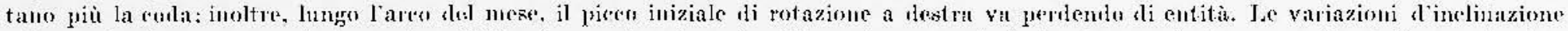

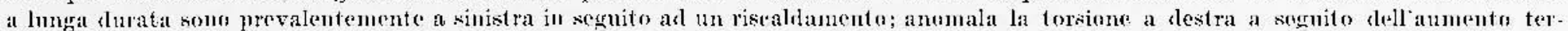
mico nej primi ginmi del mese. Ja rompenente monte-valle si presenta, al solito, in perfetto paraledismo con landanemto termiso, sia a brese slee a lungo periulu. 


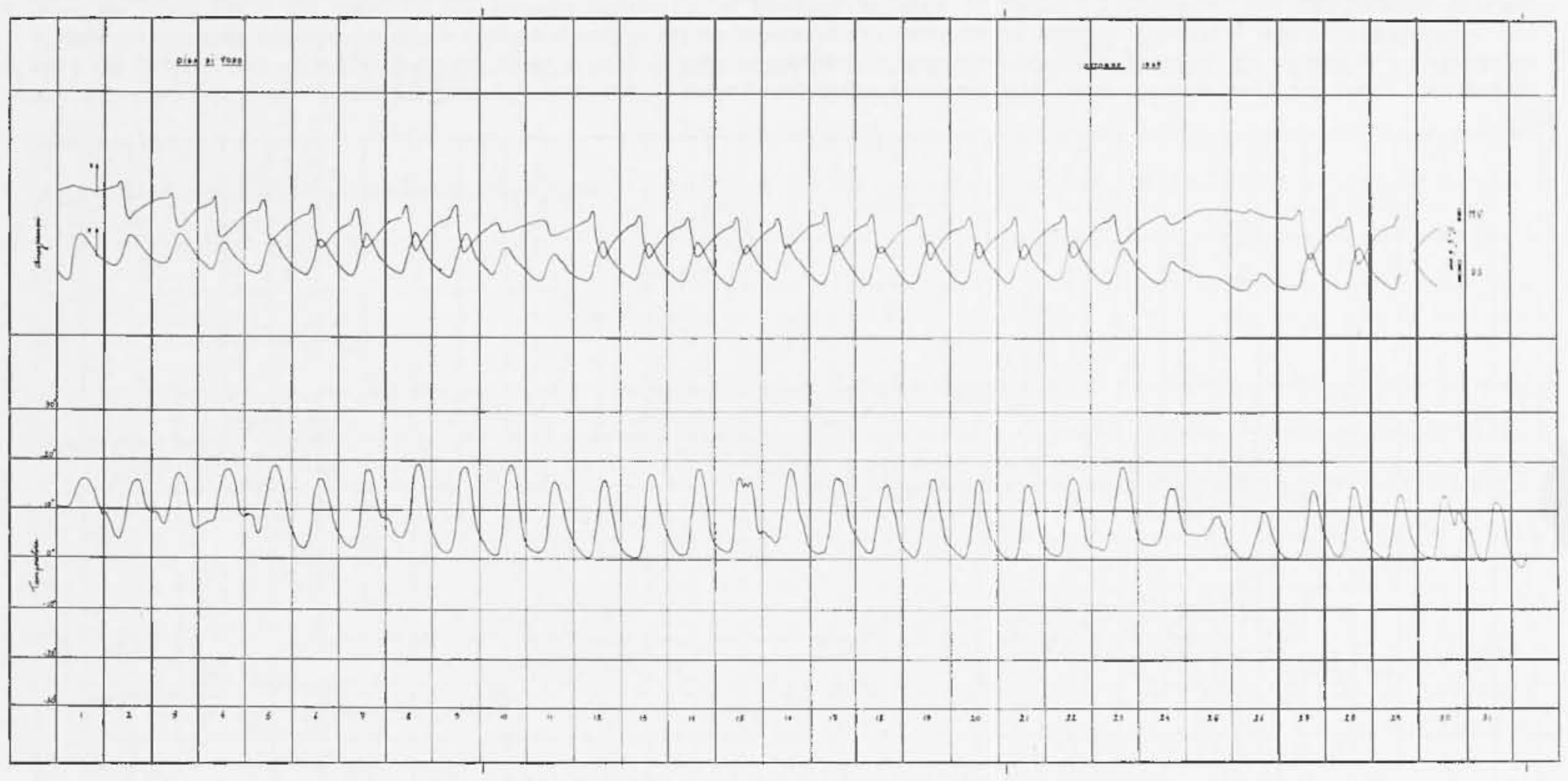

Fig. 12 - Dirra di Vodo - Ottobre 1969 - Scarsi contrasti nei elinogrammi,esgendo il diagramuma termico poco frastagliato. Le onde diurne della componente sinistra-rlestra non presentano affatto la lcoda; il piceo di iniziale rotazione a destra e alquanto piccolo rispetto alla susseguente fase di rotazione a sinistra. 


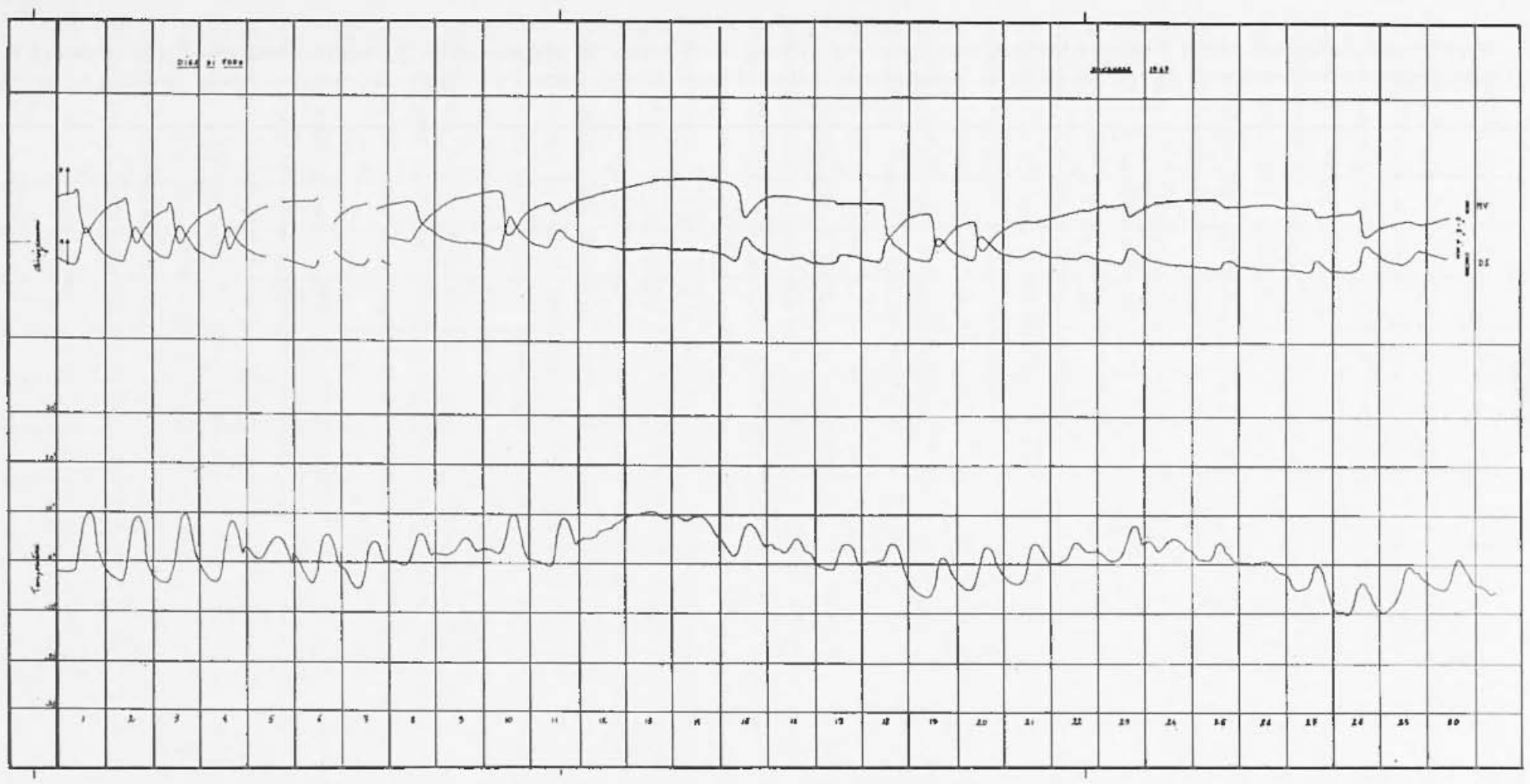

Fig. 13 - Diga di Vodo - Novembre 1969 . Je onde diurne sono presenti in molo netto, in entrambe le componenti, nolo nei gimmi di maggior escursiono termica. L'oscillazione diuma della componente sinistra-ilestra, dopo i prini gioni del mese non presenta più il pieco iniziale al rotazione a destra. Le variazioni di inclinazione a lungo periolo si presentano in modo nettamente anomalo urla componente monte-valle; si rileriuno infatti flesuioni a valle a seguito di um risealdamento e a monte per raffrethlamento. Nella conponente sinistra-destra si banno variazioni d'iuclinazione a lungo periodo a slestra per risealdanento. 


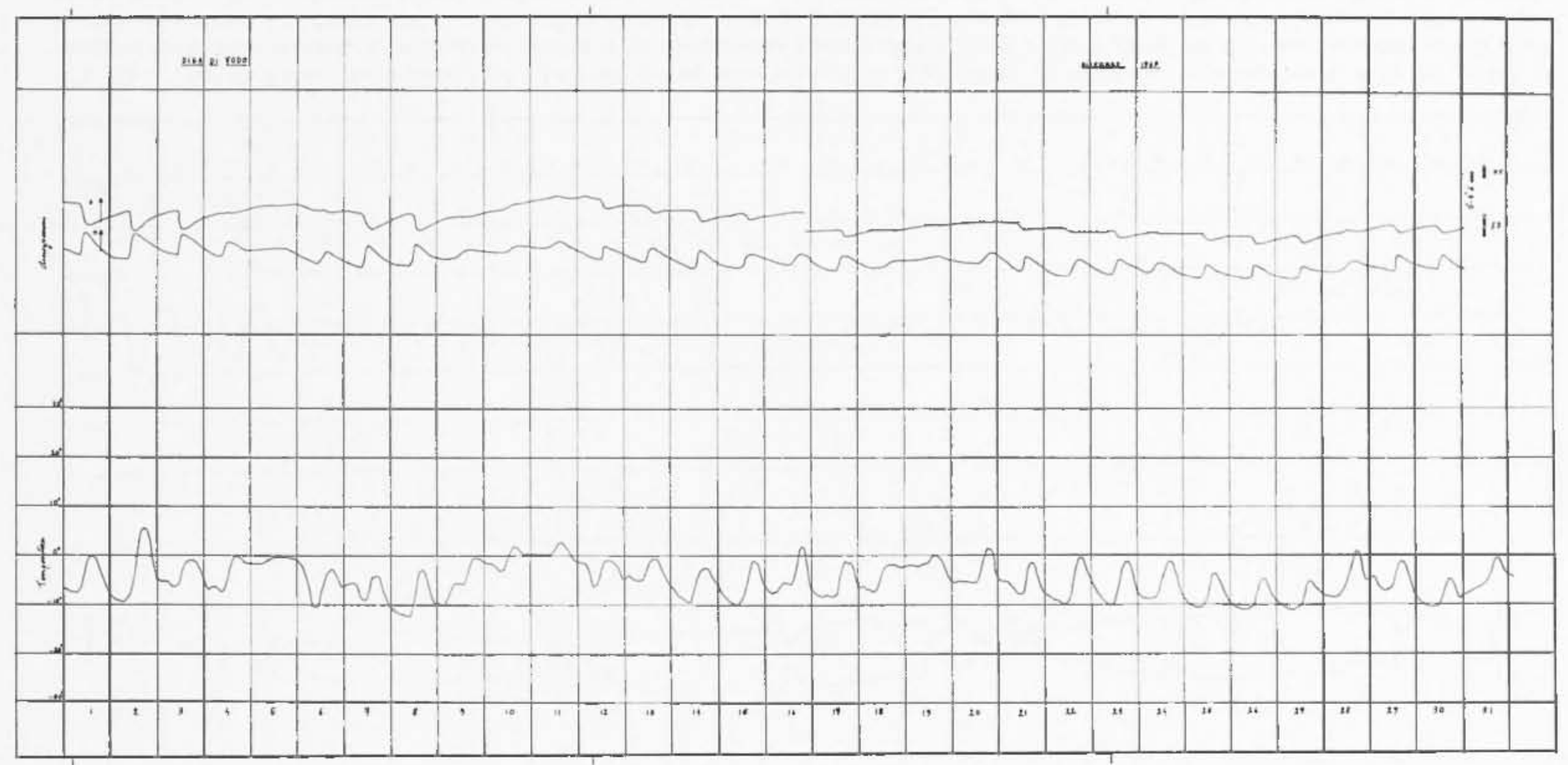

Fig. 14 - Diga di Folo - Dicembre 1969 . Le onde diume sono presenti in entrambe le componenti. II elinogramme sinistrat-destra non presenta nelloscillazione diurna il piceo iniziale di rotazione a destra. Le variazioni dinelinazione a Iungo periodo sono a destra e a monte per risealdamento 
durante tutto il 1969 , si puo infatti notare la presenza, in entrambe le componenti, di nette onde diune, in tutti i mesi.

Le ondo diurne in direzione monte-valle lawmo lib lono origine, come si è osservato per altre digho, nella dilatiazione o oonsoguente flessione vorso monte dello sbarramento durante loinsolazione, il fonomeno contrario verilicandosi al insolazione ultimatio.

Una particolare attenzione meritano invece le onde diume in dire-

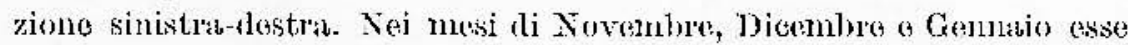
rivelano una flessiono verso sinistra durante linsolazione ed una consegueste a destra nella fase di raffreddamento. Da Febbraio a Ottobre

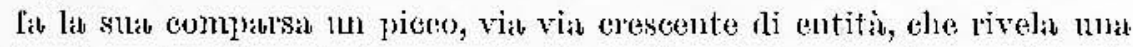
flessione iniziale a destra mentre da Aprilo al Agosto viene registrata vioppiì una ooda, sintomo di un'ulteriore flessione a sinistrab an insoarione apprabentemento nolimratias.

Le risultanti del moto dolla diga, soggrottat agli elfetti dell'insolazione, illustrano ancora moglio la sithazione rilevata. Si ossorvino

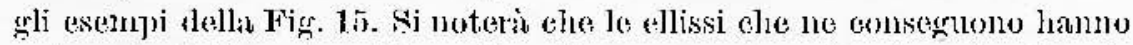
nei mesi di Geminio e Nowembre, l'asse magrsiore diretto nel primo (e

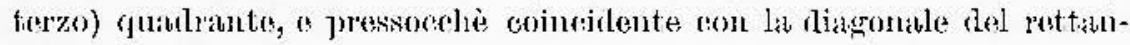
golo circoseritto, che ha per thrnensioni lo ampiezze doi moti componenti, mentre nei mesi ali Aprile "Lughio le ellissi sono riferite andi ansi coordinati. Questo vuol dire che la differenza di fasce delle vibrazioni omponenti è circa 0 nel primo caso e cirea $x / 2$ nel socondo. Quello che provopa il passagrgio della difterenza di fase dei noti componenti dabl valore 0 al valore $\pi / 2$ d appunto la eomplarsa, nella momponomte sinistrab destra, del piceo e dellib corla su citati. Nej mesi di Hamo

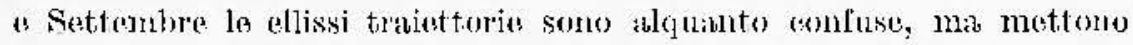
in evidenza pur sempre lat compariab di uno sfatsmento rispetto at quelle di Gemaio e Novembre, sfistumento who è provociato dalla eomprarsa, nella componente sinistra destra, del solo piceos.

I fatti osservati trovano una loro spiegabione rigorosib, se si tiene conto delle zone interessate dall'insolazione nelle varie ore dei divessi mesi.

Le onde diume della componente sinistra-rlestra nei mosi di Novenbro, Dicombro a Gentaio sono così da at tribuirsi all asimmetria dell'insolazione, insolazione ohe in questi mosi indugiab molto a lungo sul bato sinistro dellaw diga, anche quando il lato destro is gria in ombla (lab situazione è identica a quella elue si manifesta, lungo l'arco dell'suno interesrasto dall'insolazione, presso la diga dell'Ambiesta). Si vedauno gli exempi della Fig. 16. Il piceo daj mesi dab Fobbrabo ad Ottobre trova invece lab 

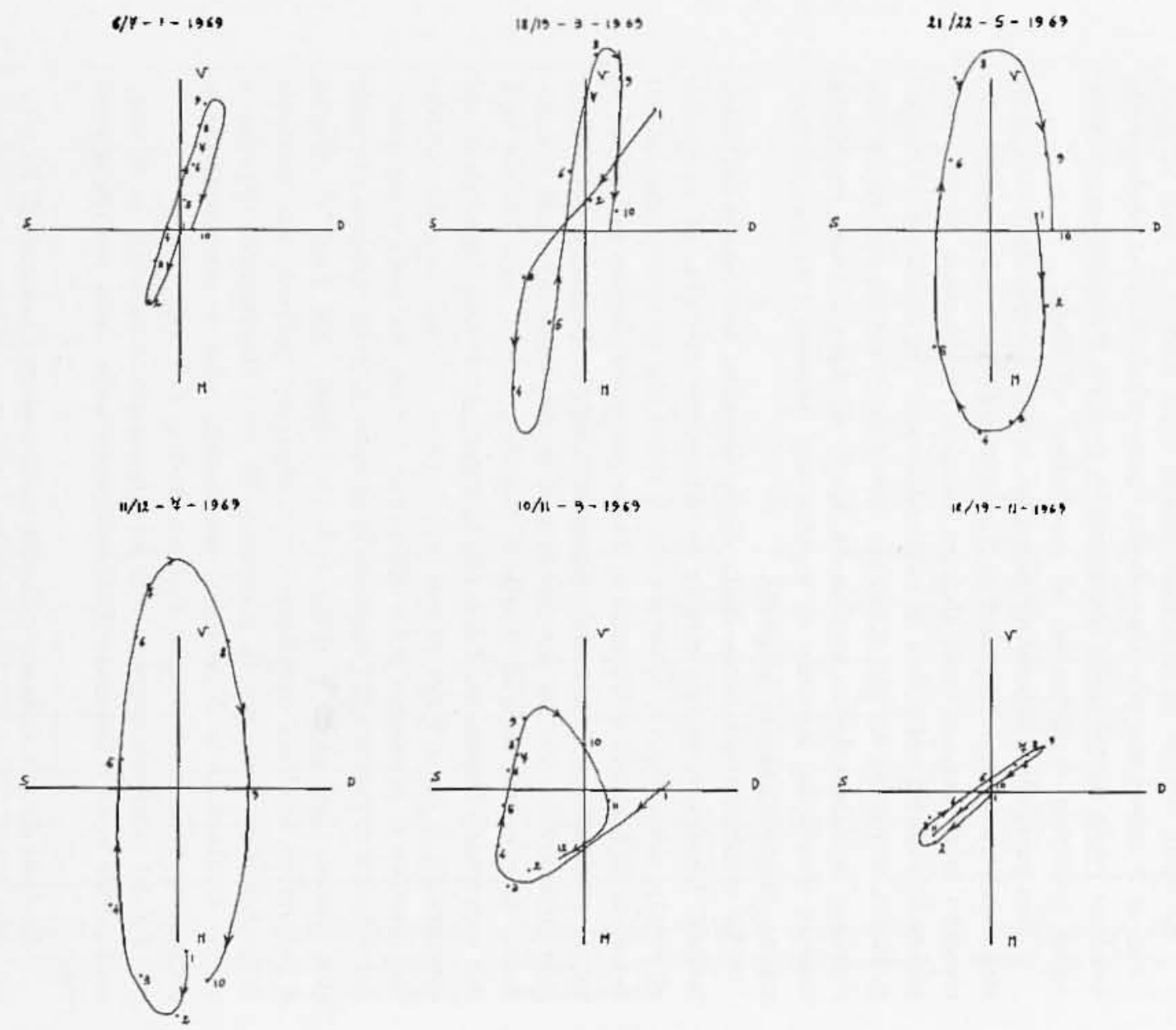

Fig. 15 - Diga di Vodo - Esempi di composivione dei movimenti diumi della diga. 
sua rayion d'essere nel soffermarsi dell'insolazione, nella prime ore del giomo, sul lato destro della diga con una duratis via via crescente (Fir. 17a). Isa dilatazione longitudinale conseguente detemina la notata flessione della diga ar destra.

10

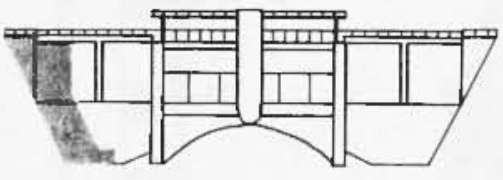

11

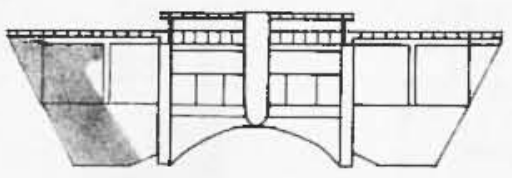

13
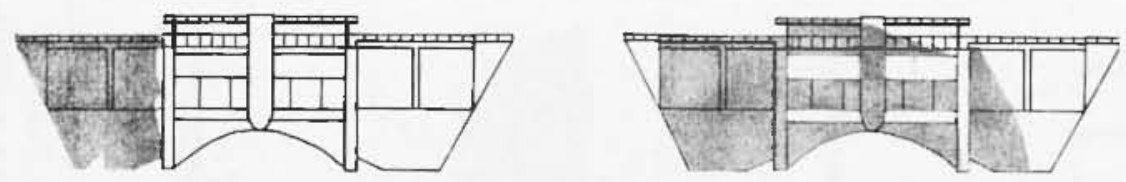

Fig. 16 - Diga di Vodo - Zone illuminate daI sole nelle ore del giorno into. ressato dall'insolazione (20 Novembre 1968 stexondo l'autore).

Per quanto eoncerne la coda che appare da Aprile ad Agosto essa risulta eollegata a quanto segue: l'insolaziono, in questi mesi, inverte il suo raggio d'azione durante il giorno, l'issolazione passa ció dalla parte della diga rivolta a valle alla parte della diga rivolta a moute. Essa indugia peró sempre sul lato sinistro, come si può rilevare dagli esempi della Figr. 17b. Al momento dell'inversione suddetta inizia una ulteriore flessione a sinistra della diga, rappresentata dalla coda, che termina ad insolazione ultimata.

Si noti che quasudo l'insolazione passa a monte, si ha un nlteriore contributo alla lissione a valle delle digu, oltre a quello causato dalla contrazione del calcestruzzo della parete a valie dello sbarramento.

E passiamo all'ultimo punto.

Le variazioni di temperatura media lnugo l'arco di un mese sono causa di micromovimenti della diga. Un aumento di temperatura media determina flessioni lunghe della diga verso monte.

Caratteristico è inoltre i) comportamento delia componente sinistra-destra, in cui a seguito di un aumento termico si registrano flessioni lungle, ora a destra ora a sinistra. Un attento csame chiarisce la situazione messa ora in evidenza. 


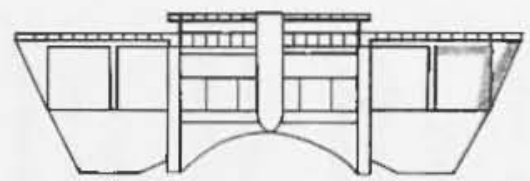

II

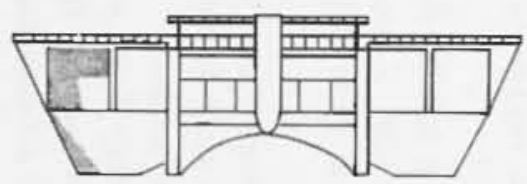

15
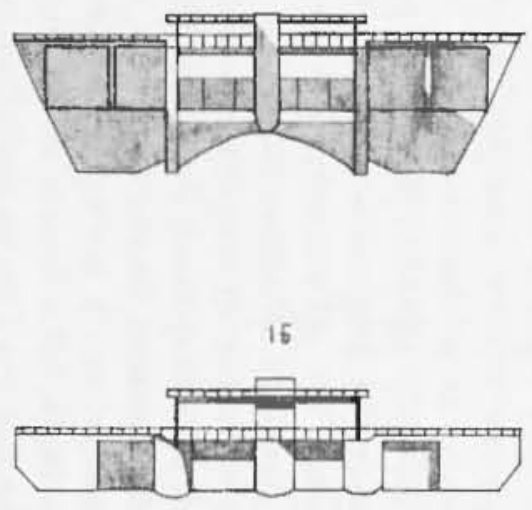

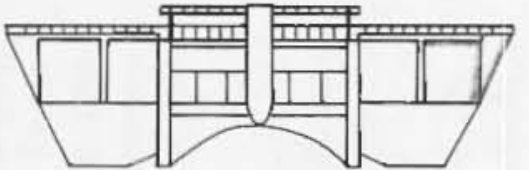

$14-x-1968$

(a)

12

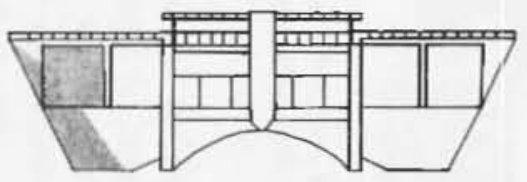

16

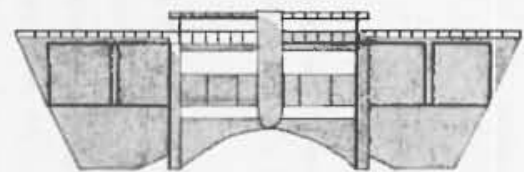

$23-14-1969$

(b)

16

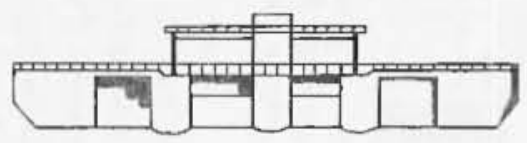

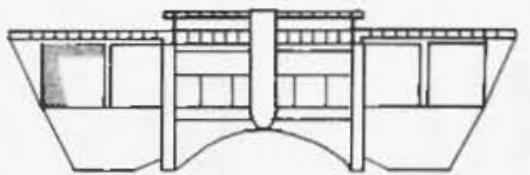

13

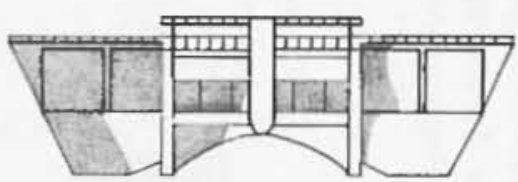

17

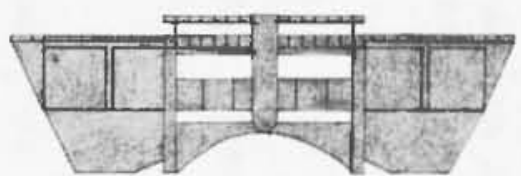

$\underline{5}$

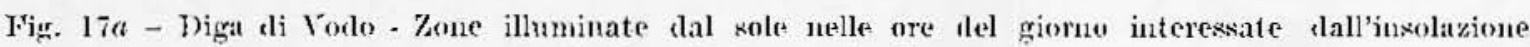
(1+ Ottobre joljs secondlo lautore).

Fig. $17 b$ - Diga di Vodo - Zone illuminate dal sole, a valle e a monte, nelle ore del giorno interessate dall'insolazione (23 Aprile 1969 secondo l'antore). 
Si ossorvi la Fig. 18: al risoaldamento di eui in (a), la diga risponde con una flessione totale a sinistra (b) se la giornata è limpiola, mentre se il sole si offusea nel pomeriggio si ha una contraziono dolla sbarramento ed una flessione totale a destra (c) (mancanza dolla soconda coda).

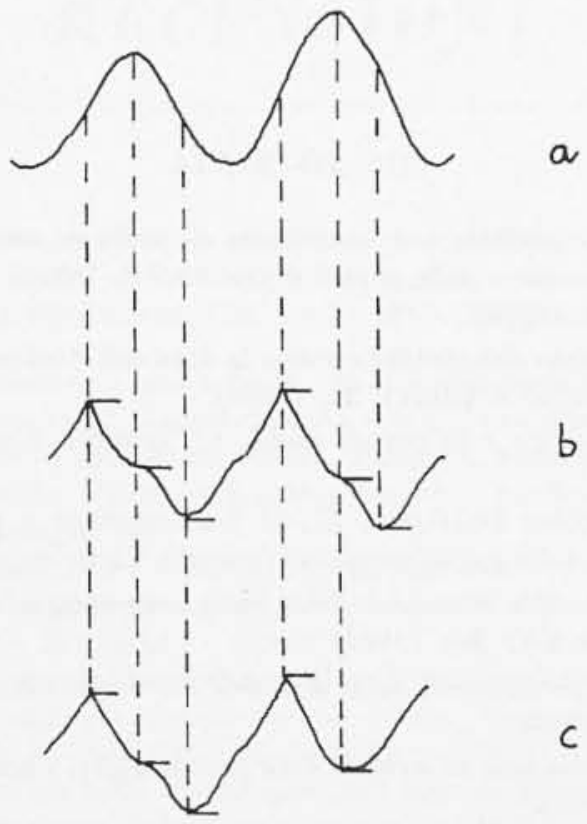

Fig. 18 - Diga di Vodo - a) esempio di anmento elella temperatura media; b) risposta (clla eomponente sinistra-slestra all'aumento di cui in a), nellipotesi (mesi da Aprile and Agosto) ele l'insolazione perturi ancle quando il suo raggio el'azione passa dalla parte a monte dolla sliga; c) risposta della componente sinistra-1estra all'aumento cli cui in a), nell'ipotesi che l'insolazione cessi di agire, passando a monte della diga.

Ia situazione illustrata non è invero generale: ossas si arlatta infatti ad una situazione particolare, nei mesi da Aprile ad $\Lambda$ gosto. Na il tipo di ragionanento $\dot{~ g e n e r a l c, ~ n e l ~ s e n s o ~ c h e ~ g l i ~ s p o s t a m e n t i ~ a ~ l u n g a ~ d u r a t s ~}$ della diga vengono ad ossere legati alle condizioni metereologiche nel corso dei giomi in oui si verificano variazioni di temperatura media; ciò spiega il singolare dualismo rilevato dai clinogrammi.

Ci si potrebbo eliedore il perché non si la un fenomono analogo sulla componente monte-valle. In effotti, in direzione monte-valle, 
causa dei micromorimenti deve considorarsi la variaziono della temporatura conseguente all'evolversi dell'insolazione, montre in direzione sinistra-destra i contrasti che danno origine ai micromovimenti nascono dall'insolazione stessa, con la sua azione asimmetrica e questo spiega il perché solamonto la componente sinistra-destra presenti delle variazioni d'inclinaziono, strettamente legate al perdurare dell'insolazione durante il giorno.

\section{BIBLIOG RAFIA}

Calor P., Come la Geofisica pus conlribuive ai problemi concernenti la costruzione e l'osservazione delle grandi dighe. SADE, Ufficio Studi, "Relazione o Studi s, 19, (1958).

CALOI P., Osservanioni olinografiche presso la diga dell' Ambiesta. SADE, Ufficio Studi, "Relazioni o Studi ", 21, (1959).

CaLor P., La Geofisica e le grandi dighe. "L'Lnnergia Llettrica», XXXIX, I, (1962).

CaLoi P. - De Rossi Diamani Ii., Il jotoclinografo a pendolo orizzontale nella teoria e nella applicazione. "L'energia Eloitrica ", XXXII, (1956).

$\mathrm{C}_{\Delta \mathrm{LOI}} \mathrm{P} .$, Aspelti della dinamica delle roce, calcestruzzo ed acque. "Annali (i) Geofisica "XV, 2-3, (1962).

CaLOr P., Aspetti geodinamici della diga dell'Ambiesla. "Amali di Geofisiea s, XVII, 2, (1964).

CaLor P., La geodinamica al servizio delle grandi dighe. "Annali di Geofisiea", XVII, 4, (1964).

BraGI P. F., Olteriori indagini sul comportamento geodinamico della diga dell'Ambiesta. "Annali di Goofisica" XXII, 4, (1969). 